\title{
MARKED FATGRAPH COMPLEXES AND SURFACE AUTOMORPHISMS
}

\author{
YUSUKE KUNO, R. C. PENNER, AND VLADIMIR TURAEV \\ AbStRact. Combinatorial aspects of the Torelli-Johnson-Morita \\ theory of surface automorphisms are extended to certain subgroups \\ of the mapping class groups. These subgroups are defined relative \\ to a specified homomorphism from the fundamental group of the \\ surface onto an arbitrary group $K$. For $K$ abelian, there is a com- \\ binatorial theory akin to the classical case, for example, providing \\ an explicit cocycle representing the first Johnson homomophism \\ with target $\Lambda^{3} K$. Furthermore, the Earle class with coefficients in \\ $K$ is represented by an explicit cocyle.
}

\section{INTRODUCTION}

Consider a compact oriented surface $\Sigma$ with non-empty boundary and basepoint $* \in \partial \Sigma$. In this note, we study certain subgroups of the mapping class group $M C(\Sigma)$. These subgroups are determined by an arbitrary group $K$ and a fixed epimorphism $p$ from the fundamental group $\pi=\pi_{1}(\Sigma, *)$ onto $K$. There are two flavors to these subgroups defined by commutative diagrams

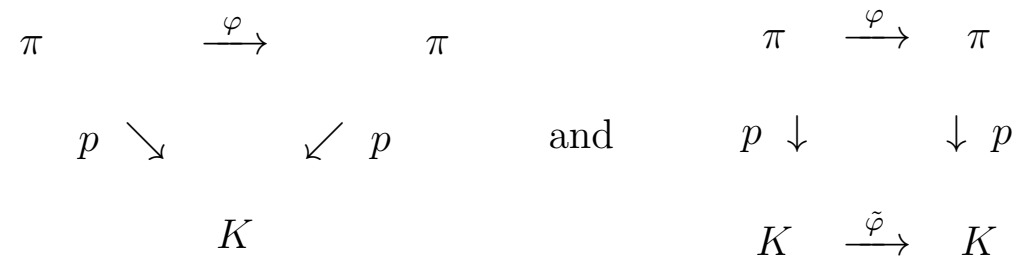

where the automorphism $\varphi$ of $\pi$ is induced by an orientation-preserving self-diffeomorphism of $\Sigma$ fixing $*$ and $\tilde{\varphi} \in \operatorname{Aut}(K)$. This leads to two respective subgroups $M C_{\nabla}(\Sigma ; p)$ and $M C_{\square}(\Sigma ; p)$ of $M C(\Sigma)$ depending upon the choice of $p$. We explain that these groups generalize, among others, the Torelli groups and the handlebody groups.

Key words and phrases. Mapping class group, Torelli group, fatgraph, Johnson homomorphism.

This work was done at the Center for Quantum Geometry of Moduli Spaces funded by the Danish National Research Foundation. 
We show that most of the combinatorial structure [1, 3, 9, 14] in terms of fatgraphs and flips coming from the cell decomposition [15] of the moduli space of $\Sigma$ persists in this more general setting.

We then focus on the case of abelian $K$. In this case, in generalization of [14], an analogue of the classical Johnson homomorphism [13] is expressed by an explicit cocycle in terms of fatgraphs whose edges are labeled by elements of $K$. Likewise, we define a combinatorial cocycle of the Ptolemy groupoid with coefficient in $K$. In the case $K=H$, the first homology of the surface, we further show that it descends to -2 times the Earle class 4 [11, which is a generator of the cohomology group $H^{1}(M C(\Sigma) ; H)$.

We emphasize the abelian case here in order to extend the combinatorial theory already developed for the mapping class groups and Torelli groups. The non-abelian case remains largely open and should be interesting to explore. Indeed, there are many open questions in this area, some of which are analogues of the classical problems while others are specific to the new technology. Several questions and problems are raised throughout the paper. For concreteness, we restrict ourselves to surfaces with one boundary component, but the general case may be addressed similarly.

\section{Notation And Background}

1.1. The Mapping Class Group. We introduce notation which will be used throughout the paper. We fix a compact oriented smooth surface $\Sigma=\Sigma_{g, 1}$ of genus $g \geq 1$ with one boundary component and fix a basepoint $* \in \partial \Sigma$. Let $\pi=\pi_{1}(\Sigma, *)$ be the fundamental group of $\Sigma$ and $\zeta \in \pi$ be the homotopy class of the loop $\partial \Sigma$ with orientation opposite to the one induced by that of $\Sigma$. Let $H=H_{1}(\Sigma ; \mathbb{Z}) \cong \mathbb{Z}^{2 g}$ be the first integral homology group of $\Sigma$. The homology intersection pairing in $H$ is skew-symmetric and non-degenerate. It is denoted ·

Let $M C(\Sigma)$ denote the mapping class group of isotopy classes of diffeomorphisms of $\Sigma$ that fix $\partial \Sigma$ pointwise. We recall the following standard facts about this group.

Fact 1.1. Any $\varphi \in M C(\Sigma)$ induces an automorphism $\varphi_{*} \in \operatorname{Aut}(\pi)$ of $\pi$ in the obvious way. The Dehn-Nielsen theorem [17] asserts that the map $\varphi \mapsto \varphi_{*}$ is an embedding of $M C(\Sigma)$ onto the subgroup of $\operatorname{Aut}(\pi)$ fixing $\zeta$. We shall identify $\varphi \in M C(\Sigma)$ with $\varphi_{*} \in \operatorname{Aut}(\pi)$.

Fact 1.2. Let $a: \pi \rightarrow H$ be the abelianization map. Any homomorphism $p$ from $\pi$ to an abelian group $K$ induces a unique homomorphism $p_{\#}: H \rightarrow K$ such that $p=p_{\#} \circ a: \pi \rightarrow K$. In particular, 
any $\varphi \in \operatorname{Aut}(\pi)$ induces an isomorphism $\varphi_{\#}: H \rightarrow H$ such that the following diagram commutes:

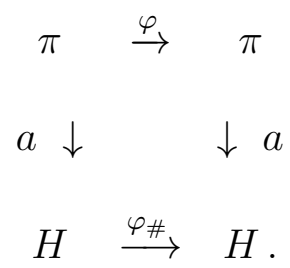

If $\varphi \in M C(\Sigma)$ (i.e., if $\varphi(\zeta)=\zeta$ ), then $\varphi_{\#}$ preserves the intersection pairing. The resulting homomorphism from $M C(\Sigma)$ to the symplectic group $S p(H)$ is an epimorphism, cf. [8].

1.2. The Torelli-Johnson-Morita theory. The lower central series $\pi=\Gamma_{0} \supset \Gamma_{1} \supset \Gamma_{2} \supset \cdots$ of $\pi$ is recursively defined by taking commutator groups $\Gamma_{k+1}=\left[\pi, \Gamma_{k}\right]$ for all $k \geq 0$. The $k$-th nilpotent quotient of $\pi$ is given by $N_{k}=\pi / \Gamma_{k}$, for $k \geq 0$. We have a short exact sequence

$$
0 \rightarrow \mathcal{L}_{k+1} \rightarrow N_{k+1} \rightarrow N_{k} \rightarrow 1,
$$

where $\mathcal{L}_{k+1}=\Gamma_{k} / \Gamma_{k+1}$ is naturally identified with the degree $k+1$ part of the free Lie algebra generated by $H$, cf. [7, 13]. Note that $N_{0}=0$ and $\mathcal{L}_{1}=N_{1}=H$.

For each $k \geq 0$, denote by $M C_{k}(\Sigma)$ the $k$-th Torelli subgroup of $M C(\Sigma)$ consisting of those mapping classes that act identically on $N_{k}$. We obtain thus the Johnson filtration

$$
M C(\Sigma)=M C_{0}(\Sigma)>M C_{1}(\Sigma)>M C_{2}(\Sigma)>\cdots
$$

of $M C(\Sigma)$. (Notation $F>G$ means in this context that $G$ is a normal subgroup of $F$.) The group $M C_{1}(\Sigma)$ is the classical Torelli group.

For each $k \geq 1$, there is a Johnson homomorphism

$$
\tau_{k}: M C_{k}(\Sigma) \rightarrow \operatorname{Hom}\left(N_{k+1}, \mathcal{L}_{k+1}\right)
$$

defined as follows. If $\varphi \in M C_{k}(\Sigma)$ and $x \in N_{k+1}$, then $\varphi(x) x^{-1} \in N_{k+1}$ projects to $0 \in N_{k}$. By the exact sequence above, $\varphi(x) x^{-1}$ is the image of a unique $y_{x} \in \mathcal{L}_{k+1}$. The formula $x \mapsto y_{x}$ defines a map $N_{k+1} \rightarrow \mathcal{L}_{k+1}$ denoted $\tau_{k}(\varphi)$. The map $\tau_{k}(\varphi): N_{k+1} \rightarrow \mathcal{L}_{k+1}$ is a homomorphism because for any $x_{1}, x_{2} \in N_{k+1}$,

$$
\varphi\left(x_{1} x_{2}\right)\left(x_{1} x_{2}\right)^{-1}=\varphi\left(x_{1}\right)\left(\varphi\left(x_{2}\right) x_{2}^{-1}\right) x_{1}^{-1}=\left(\varphi\left(x_{1}\right) x_{1}^{-1}\right)\left(\varphi\left(x_{2}\right) x_{2}^{-1}\right) .
$$

The second equality uses the fact that $\varphi\left(x_{2}\right) x_{2}^{-1}$ is central in $N_{k+1}$ as it lies in the kernel of the projection $N_{k+1} \rightarrow N_{k}$.

Claim 1.3. The map (11) is a homomorphism. 
Proof. For any $\varphi, \psi \in M C_{k}(\Sigma)$, we have

$$
\begin{aligned}
(\varphi \circ \psi(x)) x^{-1} & =\varphi(\psi(x)) x^{-1} \\
& =\varphi\left(\psi(x) x^{-1}\right) \varphi(x) x^{-1} \\
& =\psi(x) x^{-1} \varphi(x) x^{-1}=\varphi(x) x^{-1} \psi(x) x^{-1},
\end{aligned}
$$

where the last equality follows from the commutativity of $\mathcal{L}_{k+1}$ and the next-to-last equality holds because $\mathcal{L}_{k+1}$ is pointwise invariant under the automorphism of $N_{k+1}$ induced by $\varphi$. This is so because $\varphi \in$ $M C_{k}(\Sigma) \subset M C_{1}(\Sigma)$ acts as the identity in $H=\Gamma_{0} / \Gamma_{1}$ and therefore acts as the identity on all the quotients $\Gamma_{k} / \Gamma_{k+1}$.

By construction, the kernel of the Johnson homomorphism $\tau_{k}$ is $M C_{k+1}(\Sigma)$. To study the image of $\tau_{k}$, we identify

$$
\operatorname{Hom}\left(N_{k+1}, \mathcal{L}_{k+1}\right) \cong \operatorname{Hom}\left(H, \mathcal{L}_{k+1}\right) \cong H^{*} \otimes \mathcal{L}_{k+1} \cong H \otimes \mathcal{L}_{k+1}
$$

where we use the fact that $\mathcal{L}_{k+1}$ is abelian in the first isomorphism and the Poincaré duality in the last isomorphism.

Claim 1.4. [12 The image of $\tau_{k}$ in $H \otimes \mathcal{L}_{k+1}$ lies in the kernel of the additive bracket map $b_{k}: H \otimes \mathcal{L}_{k+1} \rightarrow \mathcal{L}_{k+2}$ carrying $x \otimes u$ to $[x, u]$ for all $x \in H$ and $u \in \mathcal{L}_{k+1}$.

1.3. Fatgraphs and spines. Let $G$ be a finite connected 1-dimensional CW-complex with the set of vertices $V(G)$, the set of edges $E(G)$ and the set of oriented edges $\tilde{E}(G)$. Reversing the orientation of an edge, we obtain a canonical involution $e \mapsto \bar{e}$ on $\tilde{E}(G)$. An edge of the first barycentric subdivision of $G$ is a half-edge. Given a half-edge containing a vertex $v$ of $G$, we assign the ambient edge in $E(G)$ the orientation towards $v$. In this way, we identify the set of half-edges of $G$ with $\tilde{E}(G)$.

The number of half-edges incident on a vertex of $G$ is the valence of this vertex. We shall always assume that each vertex of $G$ has valence at least three except for a single univalent vertex, whose incident edge is called the tail. The tail has a preferred orientation $t \in \tilde{E}(G)$ pointing away from its univalent endpoint. We call $G$ a fatgraph (with tail) if it is endowed with the additional structure given by a family of cyclic orderings, one such cyclic ordering on each collection of halfedges incident to a common vertex.

A fatgraph $G$ determines a compact connected oriented surface $\Sigma(G)$ as follows. For each vertex $v \in V(G)$ of valence $k$, consider an oriented polygon $P_{v}$ of $2 k$ sides, where every other consecutive side corresponds to a half-edge incident on $v$ and where the given cyclic ordering about $v$ corresponds to traversing the boundary of $P_{v}$ with the polygon on the left. The surface $\Sigma(G)$ is the quotient of the disjoint union $\sqcup_{v \in V(G)} P_{v}$, 
where the sides of polygons are identified by an orientation-reversing homeomorphism if the corresponding half-edges lie in a common edge of $G$. By the genus and the boundary number of $G$ we shall mean respectively the genus of $\Sigma(G)$ and the number of components of $\partial \Sigma(G)$. Note that if the boundary number of $G$ is equal to 1 , then there is a canonical strict order on $\tilde{E}(G)$ determined by order of appearance when traversing $\partial \Sigma(G)$ starting from the tail $t$.

A fatgraph spine of $\Sigma=\Sigma_{g, 1}$ is a pair consisting of a fatgraph (with tail) $G$ and the isotopy class relative to the boundary of an embedding $i: G \rightarrow \Sigma$ such that $i(G)$ is a strong deformation retract of $\Sigma$, the cyclic orderings about vertices of $G$ agree with the counter-clockwise sense in $\Sigma$, and $i^{-1}(\partial \Sigma)=i^{-1}(\partial \Sigma-\{*\})$ is the univalent vertex of $G$. Any such $i$ extends to an orientation-preserving homeomorphism $\Sigma(G) \approx \Sigma$. Thus, the fatgraph $G$ has genus $g$ and boundary number 1 .

1.4. Mapping Class Groupoids. The mapping class group $M C(\Sigma)$ acts without isotropy on the contractible Teichmüller space $T(\Sigma)$ of isotopy classes of hyperbolic structures with geodesic boundary on $\Sigma$. The quotient $T(\Sigma) / M C(\Sigma)$ is the Riemann moduli space of $\Sigma$, cf. [15].

Theorem 1.5. [15] For $\Sigma=\Sigma_{g, 1}$ with any $g \geq 1$, there is a $M C(\Sigma)$ invariant ideal simplicial decomposition of the Teichmüller space $T(\Sigma)$, where cells are indexed by fatgraph spines of $\Sigma$ and the face relation is given by contracting non-tail edges with distinct endpoints.

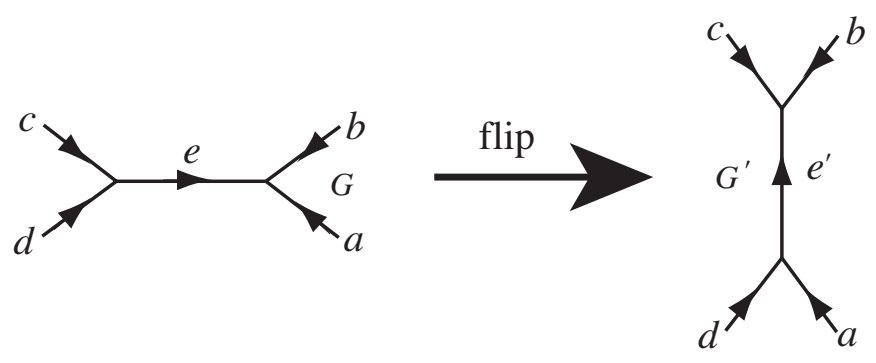

Figure 1.1. A flip

The dual of this decomposition of $T(\Sigma)$ is the dual fatgraph complex $\hat{\mathcal{G}}=\hat{\mathcal{G}}(\Sigma)$. The vertices of $\hat{\mathcal{G}}$ correspond to trivalent fatgraph spines, and the oriented 1-simplices correspond to flips, namely, the collapse and distinct expansion of a non-tail edge with distinct endpoints in a trivalent fatgraph spine, cf. Figure [1.1. Non-degenerate 2 -cells in $\hat{\mathcal{G}}$ correspond to fatgraph spines whose vertices are trivalent except for either one 5 -valent vertex or two 4 -valent vertices. 
Suppose that $G^{\prime}$ arises from $G$ by a flip along an edge $e \in E(G)$ and let $e^{\prime} \in E\left(G^{\prime}\right)$ denote the corresponding edge as in Figure 1.1. This correspondence extends to an identification of $E(G)$ with $E\left(G^{\prime}\right)$ in the obvious way. This lifts to an identification of $\tilde{E}(G)$ with $\tilde{E}\left(G^{\prime}\right)$ by demanding that the oriented edges $e, e^{\prime}$ in this order agree with the orientation of $\Sigma$ and the orientation of the other edges is unchanged as in Figure 1.1.

Recall that the fundamental path groupoid of a CW-complex $X$ is a category whose objects are the vertices of $X$ and whose morphisms are the homotopy classes of edge-paths in $X$. The Ptolemy groupoid $\operatorname{Pt}(\Sigma)$ of $\Sigma$ is the fundamental path groupoid of $\hat{\mathcal{G}}(\Sigma)$.

Corollary 1.6. [15] For any $g \geq 1$, the objects of the Ptolemy groupoid $\operatorname{Pt}(\Sigma)$ of $\Sigma=\Sigma_{g, 1}$ are the trivalent fatgraph spines of $\Sigma$, and the morphisms are finite sequences of flips subject to the following relations:

[Involutivity] the flip along an edge followed by the flip along its corresponding edge yields the identity;

[Commutativity] the flips along disjoint edges commute;

[Pentagon Relation] if two edges share a single endpoint, then the unique sequence of (five) flips along them and their corresponding edges that contains no involutive pair of flips yields the identity.

The action of $M C(\Sigma)$ on $T(\Sigma)$ induces a fixed point free action of $M C(\Sigma)$ on the $\mathrm{CW}$-complex $\hat{\mathcal{G}}$. The mapping class groupoid $M \Gamma(\Sigma)$ of $\Sigma$ is the fundamental path groupoid of the quotient CW-decomposition $\hat{\mathcal{G}}(\Sigma) / M C(\Sigma)$ of the Riemann moduli space of $\Sigma$. The objects of $M \Gamma(\Sigma)$ are the $M C(\Sigma)$-orbits of trivalent fatgraph spines of $\Sigma$ and the morphisms are generated by $M C(\Sigma)$-orbits of flips subject to $M C(\Sigma)$ orbits of the relations above. The mapping class group $M C(\Sigma)$ is isomorphic to the group of automorphisms of any object of $M \Gamma(\Sigma)$.

\section{MARKINGS}

Throughout this section we fix a group $K$.

2.1. Markings on $\pi$. A $K$-marking on $\pi=\pi_{1}(\Sigma, *)$ is an epimorphism $\pi \stackrel{p}{\rightarrow} K$. A $\nabla$-automorphism of a marking $p$ is an automorphism $\varphi$ of 
$\pi$ such that the following diagram commutes:

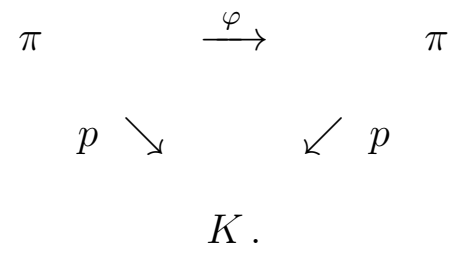

$\mathrm{A} \square$-automorphism of $p$ is an automorphism $\varphi$ of $\pi$ such that $\varphi(\operatorname{Ker} p)=$ Ker $p$. Such a $\varphi$ induces an automorphism $\tilde{\varphi}$ of $K$ making the following diagram commute:

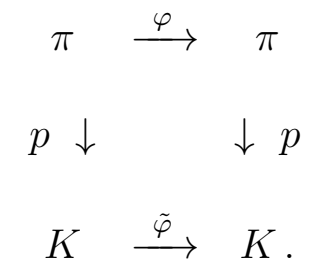

The $\nabla$-automorphisms (resp. $\square$-automorphisms) of $p$ form a group under composition, $\operatorname{Aut}_{\nabla}(p)$ (resp., $\operatorname{Aut}_{\square}(p)$ ). We have an obvious exact sequence

$$
1 \rightarrow \operatorname{Aut}_{\nabla}(p) \rightarrow \operatorname{Aut}_{\square}(p) \rightarrow \operatorname{Aut}(K) .
$$

In general, the projection $\operatorname{Aut}_{\square}(p) \rightarrow \operatorname{Aut}(K)$ is not surjective.

A $\nabla$ - or a $\square$-automorphism of $p$ is topological if it is realizable by a self-diffeomorphism of $\Sigma$ that fixes $\partial \Sigma$ pointwise. We define the p-relative $\nabla$ - and $\square$-mapping class groups of $\Sigma$ to be the respective subgroups of topological automorphisms

$$
M C_{\nabla}(\Sigma ; p)<\operatorname{Aut}_{\nabla}(p) \quad \text { and } \quad M C_{\square}(\Sigma ; p)<\operatorname{Aut}_{\square}(p) .
$$

Clearly, $M C_{\nabla}(\Sigma ; p) \leq M C(\Sigma)$ and $M C_{\square}(\Sigma ; p) \leq M C(\Sigma) \rtimes \operatorname{Aut}(K)$.

Example 2.1. If $K=(1)$, then $M C_{\square}(\Sigma ; p)=M C_{\nabla}(\Sigma ; p)=M C(\Sigma)$.

Example 2.2. For all $k \geq 1$ and the projection $\pi \stackrel{a_{k}}{\rightarrow} N_{k}$, we recover the $k$-th Torelli group $M C_{\nabla}\left(\Sigma ; a_{k}\right)=M C_{k}(\Sigma)$ while $M C_{\square}\left(\Sigma ; a_{k}\right)=$ $M C(\Sigma)$. In fact, $M C_{\nabla}(\Sigma ; p)=M C_{k}(\Sigma)$ and $M C_{\square}(\Sigma ; p)=M C(\Sigma)$ for any $N_{k}$-marking $\pi \stackrel{p}{\rightarrow} N_{k}$ because such a marking is the composition of $a_{k}$ with an automorphism of $N_{k}$. This follows from the Hopfian property of $N_{k}$, see [8], Section 5.3, Theorem 5.5.

Example 2.3. Let $F=\pi_{1}(A, *)$ where $A$ is a genus $g$ handlebody bounded by $\Sigma$ capped off by a 2-disk. If $p: \pi \rightarrow F$ is the inclusion homomorphism, then $M C_{\square}(\Sigma ; p)$ is the corresponding handlebody group relative to a disk, cf. [10], [16].

The following proposition is a direct consequence of Fact 1.2 . 
Proposition 2.4. For any abelian group $K$ and any $K$-marking $p$ on $\pi$, we have the short exact sequence

$$
1 \rightarrow M C_{\nabla}(\Sigma ; p) \rightarrow M C_{\square}(\Sigma ; p) \rightarrow I_{K}^{H} \rightarrow 1,
$$

where $I_{K}^{H}$ is the group of all $\psi \in \operatorname{Aut}(K)$ such that there is a symplectomorphism $\Psi \in S p(H)$ making the diagram

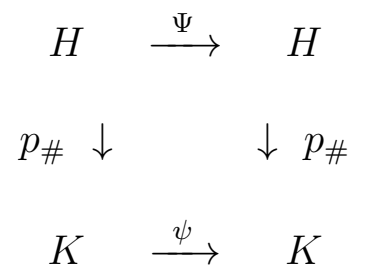

commute (here $p_{\#}$ is induced by $p$ as in Fact 1.2).

Question 2.5. Is there a better description of the group $I_{K}^{H}$ ?

Question 2.6. When are $M C_{\nabla}(\Sigma ; p)$ or $M C_{\square}(\Sigma ; p)$ finitely generated and/or finitely presented?

2.2. Markings on fatgraphs. Following [14, 3], we define a $K$-marking on a fatgraph $G$ to be a map $\mu: \tilde{E}(G) \rightarrow K$ such that

[Inversion] for any $e \in \tilde{E}(G)$, we have $\mu(\bar{e})=\mu(e)^{-1}$;

[Coherence] if $e_{1}, \ldots, e_{k}$ are the half-edges incident on a common $k$-valent vertex of $G$ in a linear order consistent with the given cyclic order, then $\mu\left(e_{1}\right) \cdots \mu\left(e_{k}\right)=1 \in K$;

[Surjectivity] the set $\{\mu(e): e \in \tilde{E}(G)\}$ generates $K$.

Example 2.7. A fatgraph spine $i: G \rightarrow \Sigma$ of $\Sigma$ determines a $\pi$ marking on $G$ as follows. Pick an oriented edge $e \in \tilde{E}(G)$ of $G$, and take a small arc $\alpha_{e}$ transverse to $e$ in $\Sigma$. We orient $\alpha_{e}$ so that the positive tangent vectors of $\alpha_{e}$ and $e$ (in this order) form a positively oriented basis in the tangent space of $\Sigma$. Since the complement of $i(G)$ is a contractible set containing $* \in \partial \Sigma$, there are unique homotopy classes of paths from the endpoints of $\alpha_{e}$ to $*$ in $\Sigma-i(G)$. These paths and $\alpha_{e}$ combine into a loop in $\Sigma$ based at $*$. The assignment of the homotopy class of this loop to $e$ determines a $\pi$-marking on $G$.

Each fatgraph spine $i: G \rightarrow \Sigma$ of $\Sigma$ determines a bijection between the $K$-markings on $\pi$ and the $K$-markings on $G$. This bijection carries a $K$-marking $\pi \stackrel{p}{\rightarrow} K$ on $\pi$ to the $K$-marking $p \mu: \tilde{E}(G) \rightarrow K$ on $G$, where $\mu: \tilde{E}(G) \rightarrow \pi$ is the $\pi$-marking derived from $i$ in Example 2.7. A $K$-marking on a fatgraph $G$ that arises in this way from a fatgraph 
spine $i: G \rightarrow \Sigma$ and a $K$-marking $\pi \stackrel{p}{\rightarrow} K$ is said to be topological with respect to $p$.

Flips along edges act naturally on marked trivalent fatgraphs. Consider a $K$-marking $\mu: \tilde{E}(G) \rightarrow K$ on a trivalent fatgraph $G$ and consider a flip along the underlying unoriented edge of an oriented non-tail edge $e \in \tilde{E}(G)$. Let $G^{\prime}$ be the resulting fatgraph with edge $e^{\prime}$ corresponding to $e$. The marking $\mu$ induces a unique $K$-marking $\mu^{\prime}$ on $G^{\prime}$ equal to $\mu$ on $\tilde{E}(G)-\{e, \bar{e}\}=\tilde{E}\left(G^{\prime}\right)-\left\{e^{\prime}, \bar{e}^{\prime}\right\}$. The coherence condition allows us to compute $\mu^{\prime}\left(e^{\prime}\right)$. In the notation of Figure 1.1,

$$
\mu\left(e^{\prime}\right)=\mu(d) \mu(a)=\mu(\bar{c}) \mu(\bar{b}) \quad \text { and } \quad \mu^{\prime}\left(\bar{e}^{\prime}\right)=\mu(\bar{a}) \mu(\bar{d})=\mu(b) \mu(c) .
$$

Observe that if the $K$-marking $\mu$ is topological with respect to $\pi \stackrel{p}{\rightarrow} K$, then so is the $K$-marking $\mu^{\prime}$.

Proposition 2.8. [3] Let $G$ be a fatgraph with genus $g$ and boundary number 1. A $\pi$-marking $\mu$ on $G$ is topological with respect to the identity map id $: \pi \rightarrow \pi$ if and only if $\mu(t)=\zeta^{-1}$ where $t \in \tilde{E}(G)$ is the tail of $G$. An $H$-marking $\mu$ on $G$ is topological with respect to the abelianization map $\pi \rightarrow H$ if and only if it respects homology intersection numbers in the sense that

$$
\mu(a) \cdot \mu(b)=\left\{\begin{aligned}
+1, & \text { if } a<b<\bar{a}<\bar{b} \text { up to cyclic permutation } \\
-1, & \text { if } a<\bar{b}<\bar{a}<b \text { up to cyclic permutation } \\
0, & \text { else }
\end{aligned}\right.
$$

where $<$ denotes the strict order on $\tilde{E}(G)$ given by occurrence along $\partial \Sigma(G)$ starting from the tail.

Proof. The first claim follows from Fact 1.1 and the Hopfian property of $\pi$. The second claim follows from the last assertion of Fact 1.2.

Question 2.9. For $k \geq 2$, when is an $N_{k}$-marking on a fatgraph topological?

\section{MARKED FATGRAPH COMPLEXES AND COCYCLES}

Fix a group $K$ and a $K$-marking $p: \pi=\pi_{1}(\Sigma, *) \rightarrow K$. Insofar as $M C(\Sigma)$ acts on the Teichmüller space $T(\Sigma)$ without fixed points, so does its subgroup $M C_{\nabla}(\Sigma ; p)$. We define the $p$-relative moduli space

$$
M_{\nabla}(\Sigma ; p)=T(\Sigma) / M C_{\nabla}(\Sigma ; p)
$$

This is an Eilenberg-MacLane space of type $K\left(M C_{\nabla}(\Sigma ; p), 1\right)$. The ideal simplicial decomposition of $T(\Sigma)$ is likewise invariant under this 
(or any) subgroup of $M C(\Sigma)$, and hence descends to the $p$-relative moduli space. The dual of this decomposition is the CW-complex

$$
\hat{M}_{\nabla}(\Sigma ; p)=\hat{\mathcal{G}}(\Sigma) / M C_{\nabla}(\Sigma ; p) \text {. }
$$

Its fundamental path groupoid is denoted by $M \Gamma_{\nabla}(\Sigma ; p)$ and called the $p$-relative mapping class groupoid. It contains $M C_{\nabla}(\Sigma ; p)$ as the stabilizer of any object.

Example 3.1. For the projection $a_{k}: \pi \rightarrow N_{k}$, the space $M_{\nabla}\left(\Sigma ; a_{k}\right)=$ $T(\Sigma) / M C_{k}(\Sigma)$ is the $k$-th Torelli space of $\Sigma$. The groupoid $M \Gamma_{\nabla}\left(\Sigma ; a_{k}\right)$ contains $M C_{k}(\Sigma)$ as a subgroup of infinite index, namely, the stabilizer of any object.

Corollary 3.2. Let $\pi \stackrel{p}{\rightarrow} K$ be a $K$-marking on $\pi$. An object of $M \Gamma_{\nabla}(\Sigma ; p)$ is given by a trivalent fatgraph with tail and a $K$-marking on this fatgraph topological with respect to $p$. Morphisms are given by finite sequences of flips acting on $K$-marked fatgraphs with tail subject to the Involutivity, Commutativity, and Pentagon Relations.

Proof. This follows from Corollary 1.6 and general position since finite sequences of flips relate any two topological $\pi$-markings on a fixed fatgraph spine with tail.

Thus, an element of $M C_{\nabla}(\Sigma ; p)$ is determined by a sequence of flips starting from a fatgraph $G$ with tail and a topological $K$-marking $\mu: \tilde{E}(G) \rightarrow K$; the sequence ends with a combinatorially isomorphic fatgraph $G^{\prime}$ with tail and a (necessarily topological) $K$-marking $\mu^{\prime}: \tilde{E}\left(G^{\prime}\right) \rightarrow K$ induced from $\mu$ by the flips so that the bijection $\tilde{E}(G) \approx \tilde{E}\left(G^{\prime}\right)$ induced by the combinatorial isomorphism $G \approx G^{\prime}$ carries $\mu$ to $\mu^{\prime}$.

Suppose now that the group $K$ is abelian. Adopting the notation of Figure 1.1 for the edges involved in a flip, we define combinatorial (untwisted) cochains

$$
m^{p} \in C^{1}(\hat{\mathcal{G}}(\Sigma) ; K), \quad j^{p} \in C^{1}\left(\hat{\mathcal{G}}(\Sigma) ; \Lambda^{3} K\right), \quad s^{p} \in C^{1}(\hat{\mathcal{G}}(\Sigma) ; K)
$$

by

$$
\begin{gathered}
m^{p}(W)=p(a)+p(c) \in K \\
j^{p}(W)=p(a) \wedge p(b) \wedge p(c) \in \Lambda^{3} K \\
s^{p}(W)= \\
([p(a) \wedge p(c)] \otimes[p(b) \wedge p(d)])+([p(b) \wedge p(d)] \otimes[p(a) \wedge p(c)]) \in S^{2} \Lambda^{2} K .
\end{gathered}
$$

Note that the obvious action of the group $M C_{\square}(\Sigma ; p)$ on $K$ induces an action of $M C_{\square}(\Sigma ; p)$ on $\Lambda^{3} K$ and $S^{2} \Lambda^{2} K$. We say that a 1 -cochain $c$ 
on $\hat{\mathcal{G}}(\Sigma)$ with values in one of these groups is $M C_{\square}(\Sigma ; p)$-equivariant if $c(\varphi(\sigma))=\varphi(c(\sigma))$ for any oriented 1-cell $\sigma$ of $\hat{\mathcal{G}}(\Sigma)$ and $\varphi \in M C_{\square}(\Sigma ; p)$.

Theorem 3.3. The cochains $m^{p}, j^{p}$, and $s^{p}$ are $M C_{\square}(\Sigma ; p)$-equivariant cocycles on $\hat{\mathcal{G}}(\Sigma)$. In particular, they descend to 1-cocycles on the cell complex $\hat{M}_{\nabla}(\Sigma ; p)$ with the corresponding coefficients.

Proof. One first checks that the cochains are well-defined, i.e., are independent of the orientation on the edge along which the flip $W$ is performed. The Commutativity Relation leads to vanishing expressions since the groups $K, \Lambda^{2} K$, and $S^{2} \Lambda^{2} K$ are abelian. Vanishing of the expressions for the Involutivity is easily proved by direct computations.

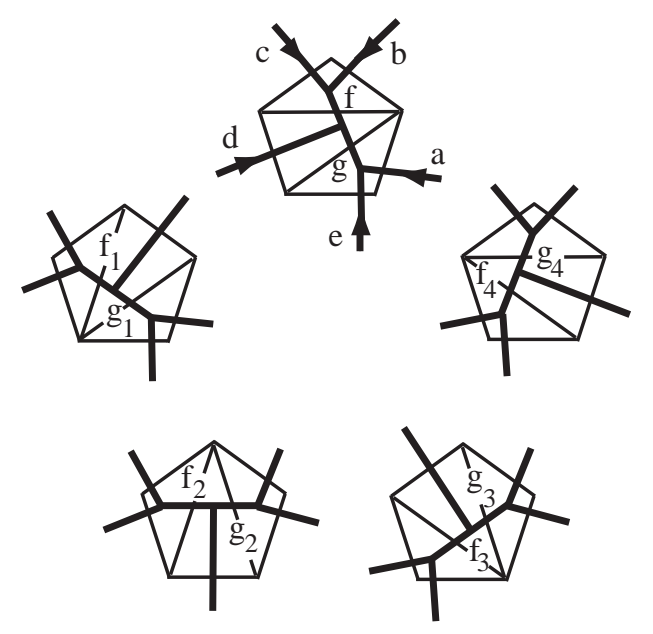

FiguRE 3.1. Notation for pentagon relation

In the notation of Figure 3.1 reproduced from [14, we shall check the Pentagon Relation $W_{f} W_{g_{1}} W_{f_{2}} W_{g_{3}} W_{f_{4}}=1$ in each case. For simplicity, we shall write $a$ instead of $p(a), b$ instead of $p(b)$, etc.

Pentagon Relation for $m=m^{p}$. We have $m\left(W_{f}\right)=b+d, m\left(W_{g_{1}}\right)=$ $b+e, m\left(W_{f_{2}}\right)=c+e, m\left(W_{g_{3}}\right)=a+c, m\left(W_{f_{4}}\right)=a+d$. Therefore,

$$
\begin{gathered}
m\left(W_{f}\right)+m\left(W_{g_{1}}\right)+m\left(W_{f_{2}}\right)+m\left(W_{g_{3}}\right)+m\left(W_{f_{4}}\right) \\
=2(a+b+c+d+e)=0
\end{gathered}
$$

as desired, where the last equality follows from Coherence.

Pentagon Relation for $j=j^{p}$ : see Theorem 3.2 of [14]. 
Pentagon Relation for $s=s^{p}$. By definition,

$$
\begin{aligned}
s\left(W_{f}\right) & =[b \wedge d] \otimes[c \wedge g]+[c \wedge g] \otimes[b \wedge d], \\
s\left(W_{g_{1}}\right) & =[e \wedge b] \otimes\left[a \wedge f_{1}\right]+\left[a \wedge f_{1}\right] \otimes[e \wedge b], \\
s\left(W_{f_{2}}\right) & =[e \wedge c] \otimes\left[g_{2} \wedge d\right]+\left[g_{2} \wedge d\right] \otimes[e \wedge c], \\
s\left(W_{g_{3}}\right) & =[a \wedge c] \otimes\left[b \wedge f_{3}\right]+\left[b \wedge f_{3}\right] \otimes[a \wedge c], \\
s\left(W_{f_{4}}\right) & =[a \wedge d] \otimes\left[g_{4} \wedge e\right]+\left[g_{4} \wedge e\right] \otimes[a \wedge d] .
\end{aligned}
$$

By Coherence, $g=a+e, f_{1}=c+d, g_{2}=a+b, f_{3}=d+e, g_{4}=b+c$.

Hence

$$
\begin{aligned}
& s\left(W_{f}\right)+s\left(W_{g_{1}}\right)+s\left(W_{f_{2}}\right)+s\left(W_{g_{3}}\right)+s\left(W_{f_{4}}\right) \\
= & {[b \wedge d] \otimes[c \wedge(a+e)]+[c \wedge(a+e)] \otimes[b \wedge d] } \\
+ & {[e \wedge b] \otimes[a \wedge(c+d)]+[a \wedge(c+d)] \otimes[e \wedge b] } \\
+ & {[e \wedge c] \otimes[(a+b) \wedge d]+[(a+b) \wedge d] \otimes[e \wedge c] } \\
+ & {[a \wedge c] \otimes[b \wedge(d+e)]+[b \wedge(d+e)] \otimes[a \wedge c] } \\
+ & {[a \wedge d] \otimes[(b+c) \wedge e]+[(b+c) \wedge e] \otimes[a \wedge d] . }
\end{aligned}
$$

Direct cancellation using the defining properties of $\wedge$ and $\otimes$ shows that the latter sum vanishes. The $M C_{\square}(\Sigma ; p)$-equivariance of the cocycles is clear.

As a consequence, the cocycles $m^{p}, j^{p}$, and $s^{p}$ give rise to twisted first cohomology classes of $M C_{\square}(\Sigma ; p)$ and untwisted cohomology classes of $M C_{\nabla}(\Sigma ; p)$. Indeed, fix a vertex $v_{0}$ of the complex $\hat{\mathcal{G}}(\Sigma)$, i.e., a fatgraph spine of $\Sigma$. For $\varphi \in M C_{\square}(\Sigma ; p)$, choose a chain $\sigma_{1}, \ldots, \sigma_{n}$ of oriented 1-cells connecting $v_{0}$ to $\varphi\left(v_{0}\right)$ and set

$$
\tilde{m}^{p}(\varphi)=\sum_{i=1}^{n} m^{p}\left(\sigma_{i}\right) .
$$

The fact that $m^{p}$ is a cocycle ensures that $\tilde{m}^{p}(\varphi)$ is well-defined. The $M C_{\square}(\Sigma ; p)$-equivariance of $m^{p}$ implies the following cocycle condition for $\tilde{m}^{p}$ : for any $\varphi, \psi \in M C_{\square}(\Sigma ; p)$, we have

$$
\tilde{m}^{p}(\varphi \psi)=\tilde{m}^{p}(\varphi)+\varphi \cdot \tilde{m}^{p}(\psi)
$$

(this construction of a twisted 1-cocycle appeared in [14], §3). The cocycle $\tilde{m}^{p}$ depends on the choice of $v_{0}$ but its cohomology classes does not depend on this choice. We obtain thus a twisted cohomology class $\left[\tilde{m}^{p}\right] \in H^{1}\left(M C_{\square}(\Sigma ; p) ; K\right)$. The cocycles $j^{p}$ and $s^{p}$ similarly give rise to twisted cohomology classes

$$
\left[\tilde{s}^{p}\right] \in H^{1}\left(M C_{\square}(\Sigma ; p) ; \Lambda^{3} K\right) \quad \text { and } \quad\left[\tilde{j}^{p}\right] \in H^{1}\left(M C_{\square}(\Sigma ; p) ; S^{2} \Lambda^{2} K\right) .
$$


These three cohomology classes restrict to respective homomorphisms

$$
M C_{\nabla}(\Sigma ; p) \rightarrow K, \quad M C_{\nabla}(\Sigma ; p) \rightarrow \Lambda^{3} K, \quad M C_{\nabla}(\Sigma ; p) \rightarrow S^{2} \Lambda^{2} K .
$$

The cocycle $m^{p}$ is new. When $p=a: \pi \rightarrow H$ is the abelianization map, the cocycle $j^{p}=j^{a}$ was discovered in [14]. It is proven in [14, 3] that $j^{a}$ represents six times the first Johnson homomorphism $\tau_{1}$. The cocycle $s^{a}$ was discovered in [15]. One can show that the cohomology class of $s^{a}$ vanishes. In $\$ 5$, we show that $\left[\tilde{m}^{a}\right] \in H^{1}(M C(\Sigma) ; H) \approx \mathbb{Z}$ is -2 times a generator called the Earle class.

Question 3.4. Are there abelian markings $p$ so that $s^{p}$ is non-trivial?

Question 3.5. What the are functorial properties in $p$ of $m^{p}, s^{p}, j^{p}$ ?

Question 3.6. For which $p$ is $j^{p}$ non-trivial? Mimicking a computation in [14], one can compute the value of $j^{p}$ on a BP torus map and find this value to be non-vanishing if a local condition holds thereby addressing this question provided the BP map lies in $M C_{\nabla}(\Sigma ; p)$.

\section{Relative Torelli-Johnson-Morita Theory}

Fix a group $K$ and a $K$-marking $\pi \stackrel{p}{\rightarrow} K$ on $\pi$. The group $K$ has its own lower central series $\Gamma_{k+1}(K)=\left[K, \Gamma_{k}(K)\right]$, where $\Gamma_{0}(K)=K$, and the nilpotent quotients $N_{k}(K)=K / \Gamma_{k}(K)$. For all $k \geq 0$, we have a short exact sequence

$$
0 \rightarrow \mathcal{L}_{k+1}(K) \rightarrow N_{k+1}(K) \rightarrow N_{k}(K) \rightarrow 1,
$$

where $\mathcal{L}_{k+1}(K)=\Gamma_{k}(K) / \Gamma_{k+1}(K)$.

Composing $\pi \stackrel{p}{\rightarrow} K$ with the projection $K \rightarrow N_{k}(K)$, we obtain a $N_{k}(K)$-marking $\pi \stackrel{p_{k}}{\longrightarrow} N_{k}(K)$ for all $k \geq 0$. The group $M C_{\nabla}\left(\Sigma ; p_{k}\right)$ plays the $p$-relative role of the $k$-th Torelli group.

Example 4.1. For $p=i d: \pi \rightarrow \pi$, we have $M C_{\nabla}\left(\Sigma ; p_{k}\right)=M C_{\nabla}\left(\Sigma ; a_{k}\right)$ where $a_{k}: \pi \rightarrow N_{k}$ is the canonical projection.

Fix $k \geq 1$. If $\varphi \in M C_{\nabla}\left(\Sigma ; p_{k}\right)$ and $x \in \pi$, then $p_{k+1}\left(\varphi(x) x^{-1}\right) \in$ $N_{k+1}(K)$ projects to $1 \in N_{k}(K)$ and determines an element $y_{x}$ of $\mathcal{L}_{k+1}(K)$. In generalization of the classical case, the formula $\varphi \mapsto$ $\left(x \mapsto y_{x}\right)$ defines the $p$-relative $k$-th Johnson map

$$
\tau_{k}^{p}: M C_{\nabla}\left(\Sigma ; p_{k}\right) \rightarrow \operatorname{Hom}\left(H, \mathcal{L}_{k+1}(K)\right) \approx H \otimes \mathcal{L}_{k+1}(K) .
$$

In general, $\tau_{k}^{p}$ does not seem to be a homomorphism. We have the following partial result in this direction. 
Proposition 4.2. For all $k \geq 1$, the restriction of $\tau_{k}^{p}$ to the group

$$
M_{k}=M C_{\nabla}\left(\Sigma ; p_{k}\right) \cap M C_{\square}\left(\Sigma ; p_{k+1}\right) \subset M C_{\nabla}\left(\Sigma ; p_{k}\right)
$$

is a homomorphism.

Proof. For $\varphi, \psi \in M_{k}$ and $x \in \pi$ we compute

$$
\begin{aligned}
p_{k+1}\left((\varphi \circ \psi)(x) x^{-1}\right) & =p_{k+1}\left(\varphi\left(\psi(x) x^{-1}\right) \varphi(x) x^{-1}\right) \\
& =\tilde{\varphi}\left(p_{k+1}\left(\psi(x) x^{-1}\right)\right) p_{k+1}\left(\varphi(x) x^{-1}\right) \\
& =p_{k+1}\left(\psi(x) x^{-1}\right) p_{k+1}\left(\varphi(x) x^{-1}\right) \\
& =\left(\tau_{k}^{p}(\varphi)+\tau_{k}^{p}(\psi)\right)(x) .
\end{aligned}
$$

In this computation: $\tilde{\varphi}$ is the automorphism of $N_{k+1}(K)$ induced by $\varphi$; the second equality uses the inclusion $\varphi \in M C_{\square}\left(\Sigma ; p_{k+1}\right)$; and the third equality follows from the fact that $\varphi \in M_{k}$ acts trivially on $H_{1}(K) \cong$ $H_{1}\left(N_{k+1}(K)\right)$ and therefore acts trivially on $\mathcal{L}_{k+1}\left(N_{k+1}(K)\right) \cong \mathcal{L}_{k+1}(K)$ $=\Gamma_{k}(K) / \Gamma_{k+1}(K)$.

We expect a version of Claim 1.4 to hold for the map

$$
\bar{\tau}_{k}^{p}: M C_{\nabla}\left(\Sigma ; p_{k}\right) \rightarrow N_{1}(K) \otimes \mathcal{L}_{k+1}(K)
$$

obtained by composing $\tau_{k}^{p}$ with the tensor product of $p_{\#}: H \rightarrow N_{1}(K)$ and the identity on $\mathcal{L}_{k+1}(K)$. We however do not pursue this line of thought here.

Question 4.3. What is the connection between the homomorphism $M C_{\nabla}\left(\Sigma ; p_{1}\right) \rightarrow \Lambda^{3} N_{1}(K)$ produced by Theorem 3.3 and the morphism

$$
\bar{\tau}_{1}^{p}: M C_{\nabla}\left(\Sigma ; p_{1}\right) \rightarrow N_{1}(K) \otimes \mathcal{L}_{2}(K) \approx N_{1}(K) \otimes \Lambda^{2} N_{1}(K) ?
$$

Remark 4.4. The paper 3 shows that for an abelian $K$, any $K$ marking $\mu: \tilde{E}(G) \rightarrow K$ on a trivalent fatgraph $G$ with tail naturally extends to a marking $\hat{\mu}: \tilde{E}(G) \rightarrow \hat{T}(K)$ by the completed tensor algebra $\hat{T}(K)$ of $K$ so that $\hat{\mu}(e) \equiv 1+\mu(e)$ modulo terms of degree at least two for all $e \in \tilde{E}(G)$. This was done in order to obtain explicit formulae for lifts of the higher Johnson homomorphisms to the classical Torelli groupoid using Kawazumi's description [5] of these homomorphisms in terms of generalized Magnus expansions. It would be interesting to generalize these results to the $p$-relative case.

Remark 4.5. As pointed out by Jørgen Ellegaard Andersen, it is intriguing and appealing to think about $M C\left(\Sigma_{1}\right)$-markings on a fatgraph spine of $\Sigma_{2}$ as describing holonomies of $\Sigma_{1}$-bundles over $\Sigma_{2}$ in order to probe 4-manifolds. 


\section{Evaluation of the Cocycle $m^{a}$}

Let $\pi \stackrel{a}{\rightarrow} H$ be the abelianization map. The cocycle $m=m^{a}$ given in Theorem 3.3 determines a twisted cohomology class $\left[\tilde{m}^{a}\right] \in$ $H^{1}(M C(\Sigma) ; H)$. Morita [1] proved that $H^{1}(M C(\Sigma) ; H)$ is an infinite cyclic group. In this section, we show that $\left[\tilde{m}^{a}\right]$ is twice a generator.

We first review the construction by Morita [11]. Let $F(\alpha, \beta)$ be the free group of rank two, generated by $\alpha, \beta$. Any element $x \in F(\alpha, \beta)$ can be uniquely written as $x=\alpha^{\varepsilon_{1}} \beta^{\delta_{1}} \cdots \alpha^{\varepsilon_{n}} \beta^{\delta_{n}}$, where $\varepsilon_{i}, \delta_{i} \in\{0, \pm 1\}$. Set

$$
d(x):=\sum_{k=1}^{n} \varepsilon_{k} \sum_{\ell=k}^{n} \delta_{\ell}-\sum_{k=1}^{n} \delta_{k} \sum_{\ell=k+1}^{n} \varepsilon_{\ell} .
$$

Next, let $\left\{\alpha_{i}, \beta_{i}\right\}_{i=1}^{g} \subset \pi$ be the standard geometrically symplectic basis of Figure 5.2 and for $1 \leq i \leq g$, define a group homomorphism $p_{i}: \pi \rightarrow$ $F(\alpha, \beta)$ by $p_{i}\left(\alpha_{i}\right)=\alpha, p_{i}\left(\beta_{i}\right)=\beta$, and $p_{i}\left(\alpha_{j}\right)=p_{i}\left(\beta_{j}\right)=1(j \neq i)$. Using the same letter $d$, we define $d: \pi \rightarrow \mathbb{Z}$ by $d(x)=\sum_{i=1}^{g} d\left(p_{i}(x)\right)$. For $\varphi \in M C(\Sigma)$, we see that the map $\pi \rightarrow \mathbb{Z}, x \mapsto d\left(\varphi^{-1}(x)\right)-d(x)$ is a homomorphism. In this way, we get a map $\tilde{f}: M C(\Sigma) \rightarrow H$ as the composite

$$
M C(\Sigma) \rightarrow \operatorname{Hom}(\pi, \mathbb{Z})=\operatorname{Hom}(H, \mathbb{Z}) \cong H,
$$

where the last isomorphism is induced by homology intersection numbers: $H \rightarrow \operatorname{Hom}(H, \mathbb{Z}), a \mapsto(y \mapsto(a \cdot y))$. Moreover, $\tilde{f}$ satisfies the cocycle condition: $\tilde{f}(\varphi \psi)=\tilde{f}(\varphi)+\varphi \cdot \tilde{f}(\psi)$ for any $\varphi, \psi \in M C(\Sigma)$.

Morita 11] proved that for $g \geq 2$, the group $H^{1}(M C(\Sigma) ; H)$ is an infinite cyclic group generated by the class $k=[\tilde{f}]$. Actually, Earle [4] already found a cocycle representing $k$, and following [6], we call $k$ the Earle class.

Theorem 5.1. We have

$$
\left[\tilde{m}^{a}\right]=-2 k \in H^{1}(M C(\Sigma) ; H) .
$$

The minus sign in the formula comes from the difference between the convention here and that of [11], where a map $f: M C(\Sigma) \rightarrow H$ is called a twisted 1-cocycle if $f(\varphi \psi)=\psi^{-1} \cdot f(\varphi)+f(\psi)$. If $f$ is a twisted cocycle in this sense, then the map $M C(\Sigma) \rightarrow H, \varphi \mapsto f\left(\varphi^{-1}\right)$ is a twisted 1-cocycle in our sense.

To prove Theorem 5.1, we evaluate the two cocycles on a torus BP map. Let $C_{1}$ and $C_{2}$ be disjoint simple closed curves on $\Sigma$ such that their union is the boundary of a torus-minus-two-disks embedded in $\Sigma$. The mapping class $t_{C_{1}} t_{C_{2}}^{-1}$ is called a torus BP map (BP stands for bounding pair), where $t_{C_{i}}$ is the right handed Dehn twist along $C_{i}$. A torus BP map is an element of $M C_{1}(\Sigma)$. 


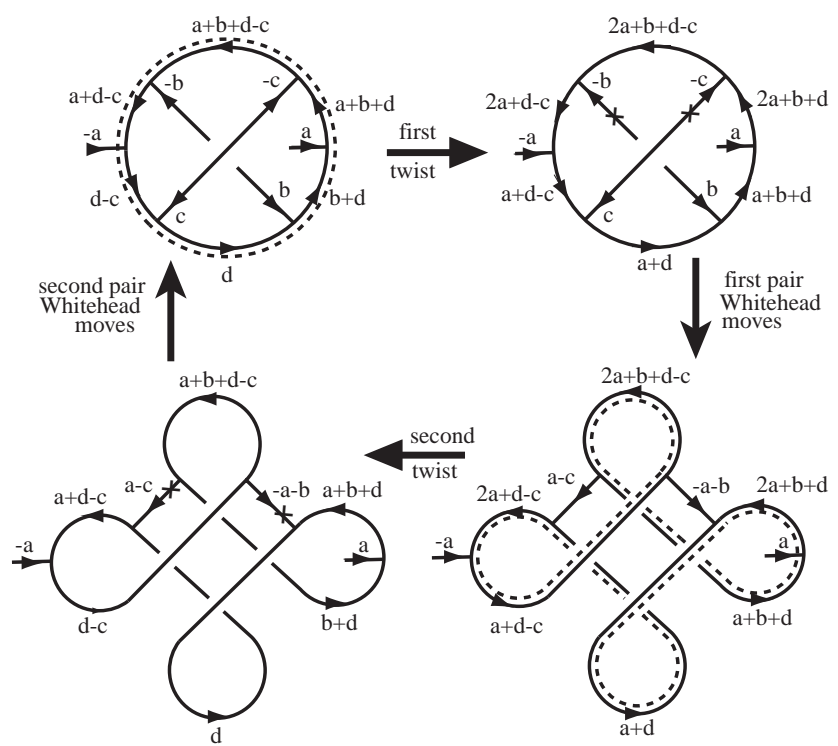

FIGURE 5.1. BP torus map expressed as flips

Lemma 5.2. Let $\varphi$ be the torus BP map illustrated in Figure 5.1 reproduced from [14]. Then $\tilde{m}^{a}(\varphi)=4 a$.

Proof. In [14], Morita and Penner expressed a torus BP map by the sequence of 14 flips in Figure 5.1, and we shall compute the contributions from each step:

(1) the first Dehn twist (5 flips):

$$
(a-c)+(a-b)+0+(a+c)+(a+b)=4 a .
$$

(2) the first pair of flips (2 flips):

$(2 a+b+d+a+d-c)+(a+d+2 a+b+d-c)=6 a+2 b-2 c+4 d$.

(3) the second Dehn twist (5 flips):

$(-a+a+b)+(-a-a+c)+0+(-a-a-b)+(-a-c+a)=-4 a$.

(4) the second pair of flips (2 flips):

$$
(-d-a-b-d+c)+(-b-d-a-d+c)=-2 a-2 b+2 c-4 d .
$$

Taking the sum, we find $\tilde{m}^{a}(\varphi)=4 a$.

For definiteness, let us choose the particular fatgraph spine of $\Sigma$ illustrated in Figure 5.3, which also depicts two simple closed curves $C_{1}, C_{2}$ and part of a homology marking viz. Figure 5.3. If $\varphi=t_{C_{1}} t_{C_{2}}^{-1}$, then $\tilde{m}^{a}(\varphi)=4 B_{2}$ in the notation of the standard basis illustrated in Figure 5.2 . 


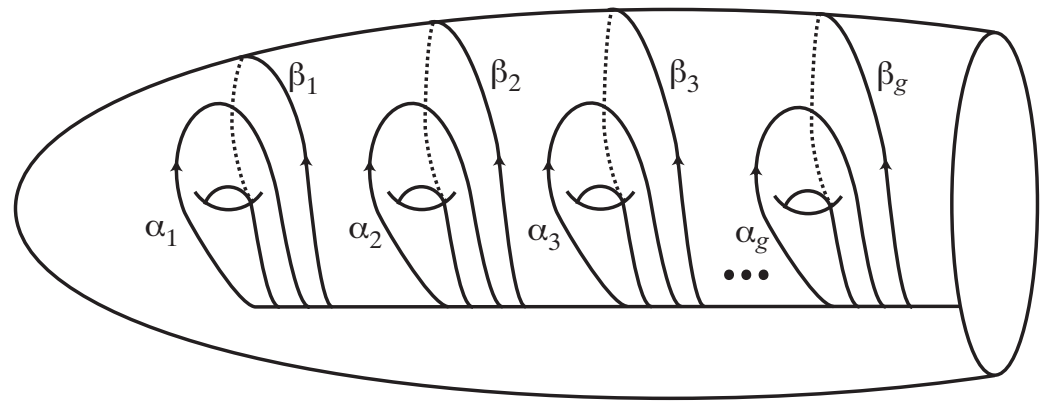

FIGURE 5.2. Geometrically symplectic basis for $\pi$

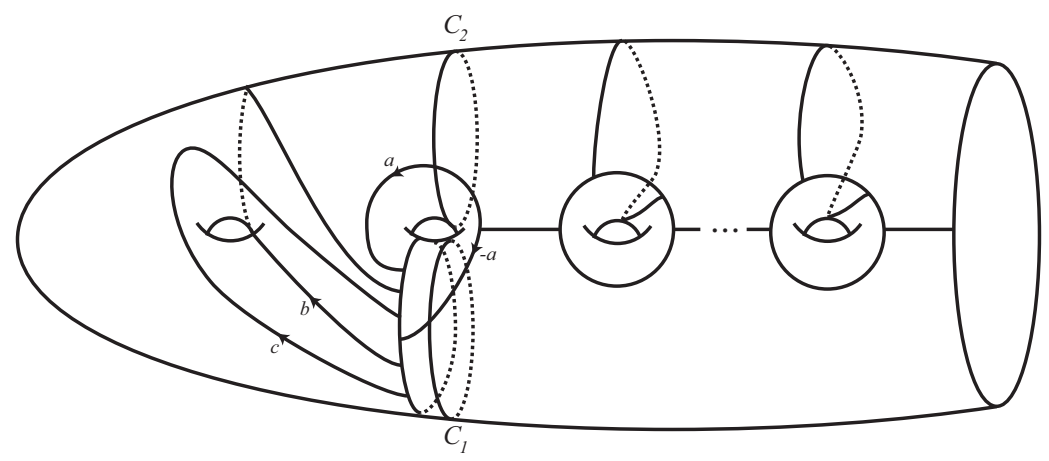

Figure 5.3. Fatgraph spine, BP map $\varphi=t_{C_{1}} t_{C_{2}}^{-1}$ and a part of homological marking

We next compute the value of $\tilde{f}$ on $\varphi=t_{C_{1}} t_{C_{2}}^{-1}$ in Figure 5.3. The action of $\varphi$ on $\pi$ is given by

$$
\varphi\left(\alpha_{1}\right)=\gamma \alpha_{1} \gamma^{-1}, \varphi\left(\beta_{1}\right)=\gamma \beta_{1} \gamma^{-1}, \varphi\left(\alpha_{2}\right)=\gamma \alpha_{2} \beta_{2}, \varphi\left(\beta_{2}\right)=\beta_{2},
$$

and $\varphi\left(\alpha_{i}\right)=\alpha_{i}, \varphi\left(\beta_{i}\right)=\beta_{i}$ for $i \geq 3$, where $\gamma=\alpha_{2} \beta_{2}^{-1} \alpha_{2}^{-1}\left[\beta_{1}, \alpha_{2}\right]$.

Lemma 5.3. Let $\varphi=t_{C_{1}} t_{C_{2}}^{-1}$ be as above. Then $\tilde{f}(\varphi)=-2 B_{2}$.

Proof. The equality $\tilde{f}(\varphi)=-\tilde{f}\left(\varphi^{-1}\right)=-2 B_{2}$ is equivalent to $d(\varphi(x))-$ $d(x)=0$ if $x \in\left\{\alpha_{i}\right\}_{i \neq 2} \cup\left\{\beta_{i}\right\}_{i}$, and $d\left(\varphi\left(\alpha_{2}\right)\right)-d\left(\alpha_{2}\right)=-2$. Since $d\left(\alpha_{i}\right)=d\left(\beta_{i}\right)=0$ for any $1 \leq i \leq g$, it is sufficient to show that $d\left(\varphi\left(\alpha_{i}\right)\right)=0$ if $i \neq 2, d\left(\varphi\left(\alpha_{2}\right)\right)=-2$, and $d\left(\varphi\left(\beta_{i}\right)\right)=0$ for $1 \leq i \leq g$. These equalities are verified by direct computations. For example, to see $d\left(\varphi\left(\alpha_{2}\right)\right)=-2$, note that $\varphi\left(\alpha_{2}\right)=\alpha_{2} \beta_{2}^{-1} \alpha_{2}^{-1}\left[\beta_{1}, \alpha_{1}\right] \alpha_{2} \beta_{2}$ so $p_{1}\left(\varphi\left(\alpha_{2}\right)\right)=\beta \alpha \beta^{-1} \alpha^{-1} \stackrel{d}{\mapsto}-2$ and $p_{2}\left(\varphi\left(\alpha_{2}\right)\right)=\alpha \stackrel{d}{\mapsto} 0$.

Theorem 5.1 follows directly from Lemmas 5.2 and 5.3 . 
Question 5.4. Can one define a counterpart of the Earle class for a general abelian $K$-marking ? What is the group $H^{1}\left(M C_{\square}(\Sigma) ; K\right)$ ?

\section{REFERENCES}

1. J. Andersen, A. Bene, R. C. Penner, Groupoid Lifts of Mapping Class Representations for Bordered Surfaces, Top. Appl. 156 (2010), 2713-2735.

2. J. E. Andersen, A. Bene, J.-B. Meilhan, R. C. Penner, Finite type invariants and fatgraphs, Adv. Math. 225 (2010), 2117-2161.

3. A. Bene, N. Kawazumi, R. C. Penner, Canonical extensions of the Johnson homomorphisms to the Torelli groupoid, Adv. Math. 221 (2009), 627-659.

4. C. J. Earle, Families of Riemann surfaces and Jacobi varieties, Ann. of Math. 107 (1978), 255-286.

5. N. Kawazumi, Cohomological aspects of Magnus expansions, preprint, math.GT/0505497(2005).

6. N. Kawazumi, Canonical 2-forms on the moduli of Riemann surfaces, in: Handbook of Teichmüller theory (A. Papadopoulos, ed. ), Volume II, EMS Publishing House, Zurich 2009, 217-237.

7. J. Labute, On the descending central series of groups with a single defining relation, J. Algebra 14 (1970), 16-23.

8. W. Magnus, A. Karrass, D. Solitar, Combinatorial Group Theory, Dover Publications, New York (2004).

9. G. Massuyeau, Canonical extensions of Morita homomorphisms to the Ptolemy groupoid. Geom. Dedicata, to appear, Doi 10.1007/s10711-011-9640$\mathrm{x}$; arXiv:1006.0920v3 [math.GT].

10. D.R. McMillan, Homeomorphisms on a solid torus, Proc. Amer. Math. Soc. 14 (1963), 386-390.

11. S. Morita, Families of Jacobian manifolds and characteristic classes of surface bundles I, Ann. Inst. Fourier 39 (1989), 777-810.

12. S. Morita, Abelian quotients of subgroups of the mapping class group of surfaces, Duke Math. J. 70 (1993) 699-726.

13. S. Morita, Structure of the mapping class groups of surfaces: a survey and a prospect, Geometry and Topology Monographs 2 (1999), Proceedings of the Kirbyfest, 349-406.

14. S. Morita and R. C. Penner, Torelli groups, extended Johnson homomorphisms, and new cycles on the moduli space of curves, Math. Proc. Camb. Phil. Soc. 144 (2008), 651-671.

15. R. C. Penner, Decorated Teichmüller Theory, QGM Masters Class Series, European Mathematical Society (2011).

16. S. Suzuki, On homeomorphisms of a 3-dimensional handlebody, Can. J. Math. 29 (1977), 111-124.

17. H. Zieschang, E. Vogt, H.-D. Coldeway, Surfaces and planar discontinuous groups, Lecture Notes in Math. 835 (1980). 
MARKED FATGRAPH COMPLEXES AND SURFACE AUTOMORPHISMS 19

Department of Mathematics, Graduate School of Science, Hiroshima University, 1-3-1 Higashi-Hiroshima, Hiroshima 739-8526, JAPAN

E-mail address: kunotti@hiroshima-u.ac.jp

Center for the Quantum Geometry of Moduli Spaces, Aarhus University, DK 8000 C Aarhus, Denmark and Departments of Mathematics and Theoretical Physics, Caltech, Pasadena CA 91125 USA

E-mail address: rpenner@imf.au.dk and rpenner@caltech.edu

Department of Mathematics, Indiana University, Bloomington IN 47405, USA

E-mail address: vtouraev@indiana.edu 